\title{
Introduction to Psychosocial Issues
}

\author{
Mark A. Chesler, Ph.D.
}

In this introduction I want to lay the groundwork for our discussions by indicating some current problems in diagnostic, treatment, and follow-up care in childhood cancer. Moreover, I want to identify some priority needs for expanded research and service. The workshop presentations and deliberations will no doubt extend and perhaps challenge some of these introductory remarks, but that indeed is our purpose in these few days.

\section{Overview}

The treatment of childhood cancer has in many ways, both physiological and psychosocial, pioneered treatment paradigms for many other forms of cancer. In fact, our researchers, medical care providers, and patients have been the ones to meaningfully introduce cancer as a "family disease" into the vocabulary. Despite these trailblazing efforts, and despite major advances in both medical and psychosocial care over several decades, six major problems in psychosocial care remain.

First, many efforts to provide psychosocial care, and to innovate new forms of care, still proceed from a "pathology" model rather than a "health" model. Most families entering into the world of childhood cancer are reasonably healthy in psychosocial terms. They are not "problem families," and seldom need psychotherapy. Rather, they are relatively normal people and families experiencing a major long-term trauma in their lives. Supportive care of various kinds, delivered in ways that respect family health, and that seek to prevent pathology, is what is needed. Unfortunately, the natural incli-

Presented at the American Cancer Society Workshop on Children with Cancer, Naples, Florida, September 5-6, 1991.

From the Department of Sociology, University of Michigan, Ann Arbor, Michigan.

In outlining the areas of need and concern, the author is indebted to the American Cancer Society-sponsored Consensus Conference, "Psycho-social Support Needs for Children with Cancer," chaired by J. van Eys, M.D., and conducted at the University of Texas M. D. Anderson Cancer Center, 1988.

Address for reprints: Mark A. Chesler, Ph.D., University of Michigan, 4016 LSA Building, Ann Arbor, MI 48109-1382.

Accepted for publication May 21, 1992. nation of the professions, the medical and psychological professions included, is to emphasize an approach to life crises that justifies and requires specialized professional resources. As life problems become "professionalized" rather than "normalized," we look for signs of pathology rather than health, of ten overemphasizing them and designing "treatment plans" (another pathology-oriented term--why not "response patterns?") on the assumption of the latter. In the case of psychosocial concerns attendant on childhood cancer, this approach not only leads to poor diagnostic assumptions (and assessment failures), but sends a message to patients and family members that often is disempowering, frightening, and may well create the very problems we seek to avoid.

Second, much psychosocial care is not conceptualized or provided on a holistic basis. That is, we too often isolate the child from the family (from parents and siblings and grandparents), and treat the child's psychosocial status and concerns without either attending to the family's concerns or seeing the child in the context of family dynamics. Moreover, even when the family is conceptualized and treated as a psychosocial unit, we all too often abstract the family from its community and neighborhood enmeshment. None of us survives alone, apart from our familial connections; and none of our families survive healthfully alone, apart from other kith and kin relations. We must plan and deliver psychosocial care in ways that deal with the entire social unit of the family-the extended family and friendship relations, and neighborhood or community contacts.

Third, psychosocial care seldom is offered on an integrated basis. That is, physicians and nurses may deal intimately with the family in the clinic or on the wards and then refer families for counseling to a stranger, perhaps a social worker in another office in another part of the hospital. One part of this treatment system may never follow up on another part, with the result often being a lack of shared information and coordination. Comprehensive assessment or treatment plans seldom are designed and implemented, and families often are shunted from office to office, from professional to professional, picking up bits and pieces of medical information, psychological insight, social support, 
and promises of help along the way. This is especially likely in large comprehensive childhood cancer treatment centers, those institutions most familiar to most of us at this workshop. This problem is due partly to a conceptual failure in the health care delivery system and partly to a general lack of coordination among medical and psychosocial specialists in large technical bureaucracies.

Fourth, psychosocial services seldom are provided over time and designed to work with a patient and family through the varied stages of medical treatment and psychosocial progress. The financial and staffing problems affecting many hospitals apparently make it infeasible to follow patients and families over time if and when they do not present "psychosocial problems." These constraints certainly make it difficult to conduct aggressive psychosocial outreach programs to families who do not present themselves. This approach to care is in marked contrast to the outreach and follow-up characteristic of the medical treatment of childhood cancer, where national research programs, local registries, national protocols, and even court cases take a very assertive outreach stance toward treatment over time.

Fifth, the psychosocial programs that are available may indeed be responsive to patient and parental concerns, but they seldom seek to empower patients and family members. Seldom are patients and families involved as partners in the design and guidance or management of these programs, and there often is substantial resistance on the part of medical and psychosocial practitioners to the notion of assertive and empowered patients and families. Seldom do staffs make an effort to share control of the treatment process with parents and patients-knowledge of treatment decisions, yes; involvement in treatment decisions, yes; but that is as far as a partnership goes ordinarily. This concern is seen in bold relief in efforts at organized and collective parent involvement in the clinic or medical system; most facilities resist such participation and empowerment, while continuing to care for and connect to families on an individual basis. One outcome of this dynamic is that many collective parental-family efforts to play a helpful role in the life of the medical care system treating their children (e.g., parent advocates/representatives on staff), or in the supportive care of other families (e.g., parent visitor or cocounseling arrangements) tend to be organized outside of the medical system.

A sixth problem is that many of the psychosocial services we know to be important, the above debates and difficulties notwithstanding, simply are not available to many patients and their families. In small community hospitals and clinics, in impoverished and oppressed communities, in financially pressed medical centers, and in hospitals or clinics serving poor popula- tions and people of color, especially, such care often is unavailable, fragmented, and poorly provided if at all.

Figure 1 presents a visual map of the several categories of people affected by childhood cancer, involved with the patient and family, and potentially beneficiaries of meaningful research and service efforts aimed at comprehensive psychosocial care. We have chosen, in the limited context of this workshop, to focus initially on five central areas of concern: 1) the patient's psychosocial health and needs; 2) the family's needs and resources and the local community setting; 3 ) the school setting and role; 4) the staff's role and impact on patient and family; and 5) terminal and bereavement care issues. We know that there is considerable overlap among these foci, and that discussion during the workshop may generate other areas of concern.

\section{Areas of Psychosocial Need and Concern}

\section{Patients}

In the care of the patient, prevention of adverse psychosocial consequences of disease and therapy, or at least minimalization of these consequences, must be recognized as an important criterion of treatment success. Some of these adverse consequences may be brought about by the physical nature of the disease and treatment, but others may evolve from the psychological trauma or pain of the disease and treatment, the fear

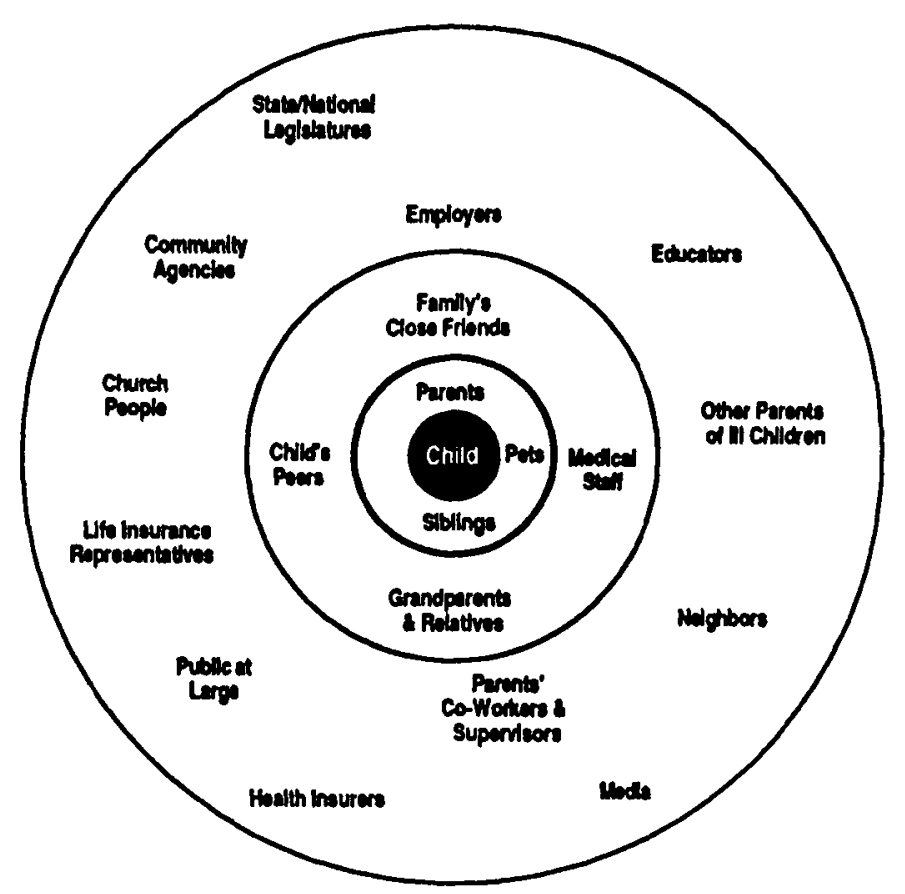

Figure 1. The widespread impact of childhood cancer. Reprinted with permission from Chesler $M$. Community support systems. In: Dowell R, Copeland D, van Eys J, editors. The child with cancer in the community. Springfield, IL: Charles C. Thomas, 1988:35. 
and worry accompanying uncertainty about the future, and the stigmatizing impact of reactions from the external social environment. Moreover, latest research indicates that many survivors of childhood cancer reflect positively on the changes and growth they have made in their lives as a consequence of positive coping with the disease. This may sound like a "pollyannish" perspective, but it is too strong and consistent a finding to be dismissed. Thus, the encouragement of positive gain, not just the prevention of negative outcomes, should be our goal.

Optimal psychosocial (or medical) care cannot be delivered unless a supportive and knowledgeable assessment plan exists. All patients differ, and the nature of these individual differences in capacities, skills, needs, and psychological styles means that any set of psychosocial support principles needs to be tuned to the individual patient. For instance, we know that many young people have an enormous and often unsatisfied desire for medical information, and the staff must be prepared to provide (and reprovide and reprovide) such information at an age-appropriate level (incidentally, this often means at a level far in advance of what the staff currently thinks is age-appropriate). Professional guidance and support should be available to patients from the outset, and opportunities for special interventions (e.g., cognitive/behavioral treatment of anticipatory nausea, self-esteem support, role-playing preparation for dealing with family and friends) should be provided to patients.

Personnel involved in creating such a total care environment and delivery system include physicians, nurses, social workers, child-life specialists, chaplains, psychologists, psychiatrists, patient/parent representatives, educators, and others with special knowledge and resources regarding childhood cancer-all working together, it is hoped, to provide care on holistic and integrated bases.

Many children with cancer report that they would have liked (or would still like) to talk with other young people (their age or perhaps a bit older) who have or have had the kind of disease and treatment they are experiencing. A peer-support system, whether organized through the hospital or clinic or through the resources of groups such as Candlelighters, would be an important response to this need.

No doubt more will be added to this introduction by colleagues Cincotta and Katz.

\section{The Family and Home Community}

The psychosocial health of the child depends, in part, on the psychosocial health of the family. In addition, since cancer affects the entire family and is now recognized as a family disease, the psychosocial health of the entire family is an important issue in and of itself. For both these reasons the entire family (parents, siblings, grandparents, and, for all we know, pets) should be involved in psychosocial assessment, support, care, and services.

For parents as well as for children, many psychosocial stresses attend this illness and its treatment, and Tabie 1 presents our own conceptualization of these stresses and their resultant coping tasks or challenges. Once again, assessment, a range of differentiated and responsive programs, and continuing provision of holistic, integrated, and empowering care is vital. Since some of the unique wisdom regarding childhood cancer is not in the hands of recognized professional experts, but is lodged in the experiential expertise of veteran parents, local Candlelighter groups become an especially important resource. Such groups are a key link in parents' efforts at self-care, self-empowerment, development of new and helpful social bonds, and active roles vis-à-vis the medical care system. How local hospital-clinics find, facilitate, respond to, and work with such groups is a matter of continuing negotiation, but an important task nonetheless. In some medical systems parent self-help and support groups have been welcomed as useful complements to the professional care provided by the staff-and as resources the staff itself can call on for help. In other medical systems the notion of parent-peer support that is unsupervised or uncontrolled by the staff generates great professional anxiety and resistance. Families should be encouraged to seek and test such peer connection and support routinely: although formal involvement may not suit the needs of all families, professional encouragement will help inform them about and legitimate these parental self-help efforts.

One of the greatest gaps in psychosocial care delivery to the family concerns siblings. Whether they show up as (extra-) "well behaved" or "acting out," siblings have strong and continuing reactions to their brother's or sister's diagnosis and treatment. The focus on caring for the patient in ways that ignore the whole family often leaves siblings bereft and neglected-in the family, in the research literature, and in the service delivery system.

We argued at the outset that childhood cancer was not only a family disease, but that all the people affected (see Fig. 1) by the illness are at potential risk (they also all are potential resources), and all should be seen as potentially involved in programs of psychosocial support and care. Obviously, caring for or mobilizing, or even contacting, this extended network is a task well beyond the current resources of most medical systems, but planning for the needs of friends and extended family members, neighbors and playmates, local commu- 
Table 1. Stresses and Coping Strategies for Parents of Children With Cancer

\begin{tabular}{|c|c|}
\hline Categories of stress & Coping strategies \\
\hline \multicolumn{2}{|l|}{ Intellectual } \\
\hline Confusion & Search for information \\
\hline Ignorance of medical terms & Search for help \\
\hline \multicolumn{2}{|l|}{ Ignorance about where things are in the hospital } \\
\hline \multicolumn{2}{|l|}{ Ignorance about who the physicians are } \\
\hline \multicolumn{2}{|c|}{ Lack of clarity about how to explain the illness to others } \\
\hline \multicolumn{2}{|l|}{ Instrumental } \\
\hline Disorder and chaos at home & Problem solving \\
\hline Financial pressures & Search for information \\
\hline Lack of time and transport to the hospital & Search for help \\
\hline \multicolumn{2}{|l|}{ Need to monitor treatments } \\
\hline \multicolumn{2}{|l|}{ Interpersonal } \\
\hline Needs of other family members & Search for help \\
\hline Friends' needs and reactions & Optimism \\
\hline Relations with the medical staff & Denial \\
\hline \multicolumn{2}{|c|}{ Behaving in public as the parent of an ill child . . . and stigma } \\
\hline \multicolumn{2}{|c|}{ Emotional } \\
\hline Shock & Denial \\
\hline Lack of sleep and nutrition & Acceptance \\
\hline Feelings of defeat, anger, fear, powerlessness & Maintenance of emotional balance \\
\hline \multirow[t]{2}{*}{ Physical or psychosomatic reactions } & Optimism \\
\hline & Reliance on religion \\
\hline \multicolumn{2}{|l|}{ Existential } \\
\hline Confusion about "why this happened to me" & Reliance on religion \\
\hline Uncertainty about the future & Acceptance \\
\hline Uncertainty about God, fate, and a "just world" & Search for information \\
\hline
\end{tabular}

nity agencies, and the like, remains an important agenda item. At the very least, educational efforts should be undertaken to instruct all involved persons (e.g., teachers, clergy, insurance and employment agencies) about the medical and psychosocial aspects of childhood cancer. With the approval of the particular family, the community support structure can be informed on an individual basis how they may assist the family. Vigorous outreach by the staff to these formal and informal community systems is an essential ingredient of a total care program. In some cases, it may be possible for medical systems or local American Cancer Society units to help create coalitions among specialized health organizations dedicated to children on a local level (some of these organizations may be formal and professional human service systems and others may be informal and voluntary organizations).

No doubt more will be added to this introduction by colleagues Kupst and Monaco.

\section{Schooling}

The maintenance of youngsters' school status and progress and their reintegration into the school environ- ment (if necessary) is of paramount importance. Schooling is the most important public extrafamily setting in which most young people learn and practice social and intellectual skills and prepare for productive occupational and socioeconomic futures. Coherent psychosocial care requires staff liaison and educational programs for school personnel and for peers of young patients. Rather than leave it to the family to bridge the gap between the medical and educational settings, it is vital that medical personnel provide local school staffs with general and individualized medical information, including recommendations for handling specific problems. Important steps in this regard are the identification and assessment of physical or neuropsychological compromises, attention to potential learning problems and stigma, proactive responses to prejudice and discrimination, and the provision of resources to deal with these issues.

Parents must be provided with information about their own and their children's legal rights to a general education, and to specific educational services, such as homebound instruction, remedial classes, tutoring services, and the like, that may be available in the local school system. Medical systems must recognize that not 
only do parents need to be informed and supported while they deal with school staffs, but that school staffs also need education and support. Most educators are ignorant of the current medical and psychosocial realities of childhood cancer. As a result, many are frightened at the prospect of having a child with cancer in the classroom. They also may feel inadequate to deal with the reactions and questions generated by the studentpatient's peers. Adequate information and liaison by the medical staff could better prepare school staffs to deal with potential problems of anxiety and awkwardness, appropriate limit-setting, peer rejection, discrimination, and alienation. Even if medical staffs are not expert in handling these problems (and there is no reason they should be), their identification and discussion of these issues may help school staffs identify and get the help they need.

In the context of schooling, an emphasis on the family nature of cancer once again draws attention to the impact on siblings of the ill child. Siblings, too, may need support in dealing with their school status and progress, and educators may need help in understanding the psychosocial stresses typically experienced by siblings of children with cancer.

No doubt more will be added to this introduction by our colleague Deasy-Spinetta.

\section{The Medical and Psychosocial Staff}

Much of the foregoing discussion has focused on things that the medical and psychosocial staff or institution should do (or consider doing) in providing adequate support and services to the patient and family members. But in addition to performing these and other functions, the staff itself is enmeshed in a series of intimate and long-term relationships with the patient and family. Care for the child and family with cancer is a high-stakes and long-term affair; people get to know one another quite well and relate quite intimately over time. As such, the patient/family-staff relationship is a very potent one.

Most of the time, of course, the staff-patient/family relationship is positive and supportive, and most patients and family members speak highly of the staffs with whom they deal. At the same time, patients and family members consistently raise a number of problematic issues about their relationships with the staff. For instance, among the concerns or problems often identified by parents are:

- Lack of adequate information

- Lack of honest and direct communication

- Inadequate resolution of conflicts

- Lack of empathy for the patient

- Lack of involvement of parents in treatment
Young patients themselves add other problems:

- Lack of sense of humor and pleasant manner

- Lack of respectful (semiadult) treatment

- Lack of real caring (over- and undersympathy)

- Lack of straight and complete information

These kinds of issues pervade any stable and ongoing human relationship, and that is what the situation is between service providers and recipients in the case of childhood cancer-people in a stable and ongoing human relationship of great import and under great stress.

These common concerns often alienate or disempower parents and young patients, and on occasion prevent them from being active participants in their child's or their own care. This is true not only in the psychosocial arena, where parents generally are experts in the care of their child, but also in the medical arena, where parents usually end up being front-line caretakers in outpatient treatment and monitoring. Well informed and actively involved parents can be a welcome complement to the medical and psychosocial staff, but only if they are prepared for these roles and if the staff genuinely invites their collaboration.

Although our focus is primarily on patients and families, and supportive services useful to them, it is clear that the medical staff also often could benefit from expanded psychosocial support. The stress of dealing with childhood cancer is substantial for health care professionals, and the rate of turnover and burnout, professional disaffection and collegial alienation, problems in patient/family-staff relations, of ten reflect this reality. Staff retreats, counseling sessions, parent representation on the staff, patient/family-staff feedback and support opportunities, all may help deal with these issues.

No doubt more will be added to this introduction by our colleague Foley.

\section{Terminal and Bereavement Care}

Special psychosocial resources must be available for the patient and family facing the terminal phase of the disease and treatment, and for the bereaved family. Whether a child endures the terminal phase of this illness in the hospital or at home (or in a hospice facility) has been a major treatment option in some medical systems, but not one available to all families. Even such options usually are implemented on medical terms alone, and there may or may not be meaningful psychosocial resources, such as an ongoing support and counseling system, provided. In addition, effective palliative care, including good pain control, is crucial at this late stage.

Ongoing support for families after the death of a 
child is a vital input to the possibilities of long-term positive coping. Even though there may not be an active patient to treat any longer, the medical system should extend psychosocial care to the bereaved family. Some support may best be forthcoming from the psychosocial staff, or it may best be available in the local self-help or Candlelighters group: parents can choose their preferred sources. Many Candlelighter groups have met with substantial success by integrating parents of living children and parents of deceased children in their programs. The parents of children living with cancer have much to learn from parents of children who have died, including overcoming some of their worst-case fears. The parents of children who have died often benefit from the knowledge that they have been able to make a positive contribution to others.

Each family should be invited to a postmortem conference, and be given the opportunity to re-review the course of the child's illness, key decisions that were made by the medical staff, and proximate reasons for death. Both individual and group counseling and support should be offered again at this time. Grief lasts a long time, and both parents and professionals need to understand the unpredictability of the duration of grief, and to gain a healthy respect for its natural course in each family.

No doubt more will be added to this introduction by our colleague Whittam.

\section{Long-Term Follow-Up}

Long-term survivors of childhood cancer, and their parents, tell us that the process of coping with childhood cancer does not end when treatment ends; nor does it end when the child is pronounced "cured" or a "longterm survivor." The memory of trauma remains for several years, as does the threat of relapse or recurrence, the need to cope with physical or psychosocial side effects, and the changed personal and familial life pat- terns and relationships engendered by the disease and consequent coping efforts. Thus, the provision of psychosocial care cannot cease with the discontinuation of therapy or the completion of the medically determined risk period.

The process of discontinuation of therapy itself often is traumatic, and patients and family members may need psychosocial assistance to prepare for and deal with this event. Anniversaries of various events (diagnosis, surgery, relapse, discontinuation) often recreate stress. Major life transitions (such as marriage, birth, geographic move, a death in the family) may also trigger memories and trauma that call for sustained attention by staffs experienced and skilled in the dynamics of the original disease and treatment process and its psychosocial concomitants.

Long-term survivors indicate a strong desire to meet and talk with others like themselves. Their desires can be supported by medical centers' efforts to create local or regional networks of survivors or by the Candlelighters agenda to create a national network of survivors of childhood cancer. In addition to providing psychosocial support and care to these young people, such a network may be a major resource in influencing public policy makers and funders, combatting societal discrimination and prejudice, and educating medical and psychosocial staffs as well as the general public.

Monitoring for medical and psychosocial (as well as socioeconomic) late effects also must be provided on a continuing basis. Late effects or long-term survivor clinics are in place at some medical centers, but not at others. For those young people who move away from the medical center at which they were originally treated, some link must be made to local internists and general practitioners who have some acquaintance with the unique medical and psychosocial concerns they carry as long-term survivors.

No doubt more will be added to this introduction by our deliberations as well as by the reports of the long-term survivor track of this workshop. 